Let us see what the effect will be when the value of $z$ as obtained from $\varrho=0$ is substituted in equations (1). Suppose that the substitution has been made in $X$ and $Z$. It is easy to see that $X_{z}$ and $Z_{z}$ are equal to zero, and that to differentiate $X$ completely with respect to $x$, it is necessary to differentiate with respect to $x$ and then to use the function of a function rule, thus $X_{x}+X_{z}(\partial z / \partial x)$, and similarly for the other letters. Thus using the fact that $\varrho=0$, we may write the equations (5) in the form

(16) $\left\{\begin{array}{c}\left(X_{p}+\frac{\partial z}{\partial p} X_{z}\right)\left(Z_{x}+p Z_{z}\right)-\left(Z_{p}+\frac{\partial z}{\partial p} Z_{z}\right)\left(X_{x}+p X_{z}\right)=0, \\ \left(P_{p}+\frac{\partial z}{\partial p} P_{z}\right)\left(X_{x}+p X_{z}\right)-\left(X_{p}+\frac{\partial z}{\partial p} X_{z}\right)\left(P_{x}+p P_{z}\right)=0, \\ \left(P_{p}+\frac{\partial z}{\partial p} P_{z}\right)\left(Z_{x}+p Z_{z}\right)-\left(Z_{p}+\frac{\partial z}{\partial p} Z_{z}\right)\left(P_{x}+p P_{z}\right)=0 .\end{array}\right.$

It is very easy to see that these equations are now the expanded form of the determinants of the matrix (15). Hence the theorem is proved.

The University of Missouri

\title{
INTEGRO-DIFFERENTIAL INVARIANTS OF ONE-PARAMETER GROUPS OF FREDHOLM TRANSFORMATIONS*
}

BY A. D. MICHAL

1. Statement of the Problem. The authort has already considered functionals of the form $f\left[y\left(\boldsymbol{\tau}_{0}^{\prime}\right), y^{\prime}\left(\boldsymbol{\tau}_{0}^{\prime}\right)\right]$ (depending only on a function $y(\boldsymbol{c})$ and its derivative $y^{\prime}(\boldsymbol{\tau})$ between 0 and 1) which are invariant under an arbitrary Volterra one-parameter group of continuous transformations. The

* Presented to the Society, December 1, 1923.

$\dagger$ Cf. Integro-differential expressions invariant under Volterra's group of transformations in a forthcoming issue of the ANNaLs of Mathematics. This paper will be referred to as "I.D. I. V." 
calculation of the invariants in question was effected in the case of a large class of functionals known as analytic functionals.

The purpose of this note is to consider the problem of finding analytic functionals $f\left[y\left(\boldsymbol{c}_{0}^{\prime}\right), y^{\prime}\left(\boldsymbol{x}_{0}^{\prime}\right)\right]$ invariant under a Fredholm group of transformations

$$
y_{1}(x)=y(x)+\int_{0}^{1} K(x, s \mid a) y(s) d s,
$$

where $a$ is the parameter of this continuous one-parameter group of transformations, and where $a=0$ corresponds to the identical transformation.

We restrict ourselves to transformations (1) for which the $y^{\prime}(\boldsymbol{\tau})$ 's exist and are continuous in the interval $I: 0 \leqq \boldsymbol{\tau} \leqq 1$; $K(x, s \mid a)$ and $\partial K / \partial x$ are continuous in $x$ and $s$ in the square $S: 0 \leqq x \leqq 1,0 \leqq s \leqq 1 ;$ and $\partial K / \partial x$ is not identically zero when $a \neq 0$.

The infinitesimal transformation corresponding to (1) will be of the form

$$
\delta y(x)=\left[\int_{0}^{1} H(x, s) y(s) d s\right] \delta a
$$

with

$$
\delta y^{\prime}(x)=\left[\int_{0}^{1} H_{1}(x, s) y(s) d s\right] \delta a,
$$

where

$$
H_{1}(x, s) \equiv \frac{\partial H(x, s)}{\partial x}
$$

as the extended group of infinitesimal transformations.

Here follow the well known relations* between the kernel $K(x, s \mid a)$ of the Fredholm finite transformation (1) and the kernel $H(x, s)$ of the corresponding infinitesimal transformation (2):

* Gerhard Kowalewski, Über Funktionenräume, Wirner SitzungsBERICHTE, 1911, vol. 120, IIA. 


$$
K(x, s \mid a)=\sum_{i=1}^{\infty} \frac{a^{i} H^{i}}{i !}
$$

(5) $H(x, s)=\left[\frac{\partial K(x, s \mid a)}{\partial a}\right]_{a=0}=\frac{1}{a} \sum_{i=1}^{\infty}(-1)^{i-1} \frac{K^{i}}{i}, a \neq 0$,

where $H^{i}$ and $K^{i}$ are to be interpreted according to Volterra's symbolic multiplication.

By methods similar to those employed in proving the lemma of Part I of I. D. I. V., we can prove* without difficulty the following lemma.

Lemma. Necessary and sufficient conditions that $H(x, s)$ and $\partial H / \partial x$ be continuous in $x$ and $s$ and that $\partial H / \partial x$ be not identically zero are that $K(x, s \mid a)$ and $\partial K / \partial x$ be continuous in $x$ and $s$ and that $\partial K / \partial x$ be not identically zero, when $a \neq 0$.

\section{A Sufficient Condition for Invariance.}

Theorem 1. Let $f\left[y\left(\boldsymbol{x}_{0}^{\prime}\right), y^{\prime}\left(\boldsymbol{x}_{0}^{\prime}\right)\right]$ be an analytic functional of $y(\boldsymbol{\tau})$ and $y^{\prime}(\boldsymbol{\tau})$, i. e., developable in a Volterra expansiont

$$
\begin{aligned}
& f_{00}+\sum_{j=1}^{\infty}\left\{\frac { 1 } { j ! } \int _ { 0 } ^ { 1 } \int _ { 0 _ { ( j , } } ^ { 1 } \ldots \int _ { 0 } ^ { 1 } \left[\sum_{k=0}^{j}\left(\begin{array}{l}
j \\
k
\end{array}\right) f_{j-k, k}\left(t_{1}, \cdots, t_{j-k} ; t_{j-k+1}, \cdots, t_{j}\right)\right.\right. \\
&\left.\left.\times \prod_{i=1}^{j-k} y\left(t_{i}\right) \prod_{i=j-k+1}^{j} y^{\prime}\left(t_{i}\right)\right] d t_{1} d t_{2} \cdots d t_{j}\right\} .
\end{aligned}
$$

We shall assume that $f_{j-k, k}$ is continuous in its $j$ arguments, symmetric separately in the sets of arguments $t_{1}, t_{2}, \ldots, t_{j-k}$ and $t_{j-k+1}, \ldots, t_{j}$ respectively; and for convenience we assume also that

$$
\left|f_{j-k, k}\right|<\gamma, \quad|y|<\varrho_{1}, \quad\left|y^{\prime}\right|<\varrho_{2},
$$

where $\gamma, \varrho_{1}, \varrho_{2}$ are positive constants. Then a sufficient condition that $f\left[y\left(\boldsymbol{\tau}_{0}^{\prime}\right), y^{\prime}\left(\boldsymbol{\tau}^{0}\right)\right]$ be invariant under a given group of transformations (1) is that it satisfy the relation

* The proof comes by a direct calculation of the series involved.

$\dagger$ This is a generalization of Taylor's series given by Volterra. See for example his Leçons sur les Équations Intégrales, 1913. 
$\int_{0}^{1} H_{1}\left(t, t_{i+k}\right) f_{i, k}\left(t_{1}, \ldots, t_{i} ; t_{i+1}, \ldots, t_{i+k-1}, t\right) d t$

$$
-\equiv \int_{0}^{1} H\left(t, t_{i+k}\right) f_{i+1, k-1}\left(t, t_{1}, \ldots, t_{i} ; t_{i+1}, \ldots, t_{i+k-1}\right) d t \text {. }
$$

The necessary and sufficient condition that $f\left[y\left(x_{0}^{\prime}\right), y^{\prime}\left(\tau_{0}\right)\right]$ be invariant under (1) is that under (2)

$$
\delta f\left[y\left(\boldsymbol{\tau}_{0}^{\prime}\right), y^{\prime}\left(\boldsymbol{\tau}_{0}^{\prime}\right)\right] \equiv 0 \text { in } y \text { and } y^{\prime} .
$$

Since the analyticity of our functionals insures the validity of a Volterra variation, we may use Volterra's* form of the variation of a functional. Then condition (9) becomes

$$
\int_{0}^{1} f_{y}(t) \delta y(t) d t+\int_{0}^{1} f_{y^{\prime}}(t) \delta y^{\prime}(t) d t \equiv 0
$$

in $y$ and $y^{\prime}$, where $f_{y}(t)$ and $f_{y^{\prime}}(t)$ are the partial functional derivatives of $f\left[y\left(\boldsymbol{\tau}_{0}^{\prime}\right), y^{\prime}\left(\boldsymbol{\tau}_{0}^{\prime}\right)\right]$ with respect to $y(\tau)$ and $y^{\prime}(\tau)$, respectively, both taken at the point $t$.

Substituting in (10) the values of $\delta y(t)$ and $\delta y^{\prime}(t)$ as given by (2) and (3), respectively, rearranging and dividing through by $\delta a$, we get

$$
\int_{0}^{1} y(s)\left[\int_{0}^{1} f_{y}(t) H(t, s) d t+\int_{0}^{1} f_{y^{\prime}}(t) H_{1}(t, s) d t\right] d s \equiv 0
$$

in $y$. We may now apply Lemma 2 of $I . D . I$. $V$; doing so, we find

$$
\int_{0}^{1} f_{y^{\prime}}(t) H_{1}(t, s) d t=-\int_{0}^{1} f_{y}(t) H(t, s) d t .
$$

Such operations as functional differentiations term by term are valid since the series involved are uniformly convergent under our hypotheses.t Calculating the partial functional derivatives $f_{y}(t)$ and $f_{y^{\prime}}(t)$, respectively, and substituting them in (12), we get by an easy reduction +

* A more general expression for $\delta f$ would be in the form of Stieltjes integrals.

$\dagger$ Cf. I. D. I. V., Part II, and Volterra's Leçons sur les Équations Intégrales, 1913, p. 18.

$\ddagger$ Cf. similar reductions of $I . D . I . V$. 
$\sum_{j=1}^{\infty}\left\{\frac{1}{(j-1) !} \int_{0}^{1} \int_{0(j)}^{1} \ldots \int_{0}^{1}\left[\sum_{l=0}^{j-1}(j-1) H_{1}(t, s) f_{j-1-l, l+1}\left(t_{1}, \ldots\right.\right.\right.$ $\left.\ldots, t_{j-1-l} ; t_{j-l}, \ldots, t_{j-1}, t\right)$

$$
\left.\left.\times \prod_{i=1}^{j-1-l} y\left(t_{i}\right) \prod_{i=j-l}^{j-1} y^{\prime}\left(t_{i}\right)\right] d t_{1} \ldots d t_{j-1} d t\right\}
$$

$$
\equiv-\sum_{j=1}^{\infty}\left\{\frac { 1 } { ( j - 1 ) ! } \int _ { 0 } ^ { 1 } \int _ { 0 _ { ( j ) } } ^ { 1 } \ldots \int _ { 0 } ^ { 1 } \left[\sum _ { k = 0 } ^ { j - 1 } ( j - 1 ) H ( t , s ) f _ { j - k , k } \left(t, t_{1}, \ldots\right.\right.\right.
$$

$$
\begin{aligned}
\ldots, t_{j-k-1} ; t_{j-k}, & \left.\ldots, t_{j-1}\right) \\
& \left.\left.\times \prod_{i=1}^{j-k-1} y\left(t_{i}\right) \prod_{i=j-k}^{j-1} y^{\prime}\left(t_{i}\right)\right] d t_{1} \ldots d t_{j-1} d t\right\},
\end{aligned}
$$

where $k=l+1$. Equating coefficients of similar terms in $y$ and $y^{\prime}$, we find

$$
\begin{aligned}
& \int_{0}^{1} H_{1}(t, s) f_{j-1-l, l+1}\left(t_{1}, \ldots, t_{j-1-l} ; t_{j-l}, \ldots, t_{j-1}, t\right) d t \\
& \quad \equiv-\int_{0}^{1} H(t, s) f_{j-l, l}\left(t, t_{1}, \ldots, t_{j-1-l} ; t_{j-l}, \ldots, t_{j-1}\right) d t
\end{aligned}
$$

which can be written in the form (8).

3. Calculation of the Invariants $f\left[y\left(\boldsymbol{\tau}_{0}^{\prime}\right), y^{\prime}\left(\boldsymbol{\tau}_{0}^{\prime}\right)\right]$. In order that $f\left[y\left(\boldsymbol{\tau}_{0}^{\prime}\right), y^{\prime}\left(\boldsymbol{\tau}_{0}^{\prime}\right)\right]$ be invariant under (1) it is sufficient that the following recurrence formula hold

$$
\equiv-\frac{H\left(t, t_{i+k}\right)}{H_{1}\left(t, t_{i+k}\right)} f_{i+1, k-1}\left(t, t_{1} \ldots, t_{i} ; t_{i+1}, \ldots, t_{i+k-1}\right) \text {. }
$$

We shall now prove the following theorem.

Theorem II. A necessary and sufficient condition on (1) that an analytic functional $f\left[y\left(\boldsymbol{\tau}_{0}\right), y^{\prime}\left(\boldsymbol{\tau}_{0}^{\prime}\right)\right]$ be invariant under (1) when (14) holds is that the kernel $H(x, s)$ of the infinitesimal transformation be of the form

$$
H(x, s) \equiv \psi(s) e^{x / c},
$$


where $\psi(s)$ is an arbitrary function of $s$, and where $c$ is a constant.*

It is evident from (14) that

$$
\frac{H\left(t, t_{i+k}\right)}{H_{1}\left(t, t_{i+k}\right)}
$$

must be independent of $t_{i+k}$, and hence it is necessary that it be a function of $t$ alone, say $\varphi(t)$. On applying (14) until $f_{i, k}$ is written in terms of $f$ 's with second index zero, we get the recurrence formula

$$
\begin{aligned}
& f_{i, k}\left(t_{1}, t_{2}, \ldots, t_{i} ; t_{i+1}, \ldots, t_{i+k-1}, t\right) \\
= & (-1)^{k}[\varphi(t)]^{k} f_{i+k, 0}\left(t, t_{1}, \ldots, t_{i}, t_{i+1}, \ldots, t_{i+k-1}\right) .
\end{aligned}
$$

By hypothesis $f_{i+k, 0}$ is symmetric in all its arguments. Therefore, interchanging $t_{1}$ and $t_{i+1}$ leaves the right-hand side of (16) unchanged. Hence if (16) is to hold, $f_{i, k}$ must be symmetric with respect to $t_{1}$ and $t_{i+1}$, and therefore it must be symmetric in all its arguments. On interchanging $t$ and any $t_{j}$ in (16), we see at once that $\varphi(t)$ must be a constant, say $c$; i. e., $H(x, s)$ must satisfy the equation

$$
c \frac{\partial H(x, s)}{\partial x}-H(x, s)=0,
$$

whose most general solution is (15).

We may now remark that the arbitrariness of the coefficients $f_{i+k, 0}$, in terms of which all the other $f_{i, k}$ 's can be evaluated, on making use of the recurrence formula, enables us to state immediately the following theorem.

THEOREM III. Let the kernel $H(x, s)$ of the infinitesimal transformation (2) be of the form $\psi(s) e^{x / c}$, and let us take an analytic functional $f\left[y\left(\boldsymbol{\tau}_{0}^{\prime}\right), y^{\prime}\left(\boldsymbol{x}_{0}^{\prime}\right)\right]$, all of whose $f_{i, k^{\prime}}$ 's are symmetric in all their arguments, and assign arbitrarily for initial conditions the coefficients $f_{i+k, 0}$ in its Volterra expansion; that is, take an arbitrary $F\left[y\left(\boldsymbol{x}_{0}^{\prime}\right)\right]$ such that $F\left[y\left(\boldsymbol{\tau}_{0}^{\prime}\right)\right]$ $\equiv f\left[y\left(\boldsymbol{r}_{0}^{\prime}\right), y_{0}^{\prime}\left(\boldsymbol{\tau}_{0}^{\prime}\right)\right]$, and for convenience take $y_{0}^{\prime}(\boldsymbol{\tau}) \equiv 0$. Then, if the $f_{i, k}$ 's are calculated by the recurrence formula

* That is, if $H(x, s) \equiv \psi(s) e^{x / c}$, we may assert that invariant analytic functionals $f\left[y\left(\tau_{0}^{\prime}\right) y^{\prime}\left(\tau_{0}^{\prime}\right)\right]$ always exist. 
(18) $f_{i, k}\left(t_{1}, \ldots, t_{i+k}\right)=(-1)^{k} c^{k} f_{i+k, 0}\left(t_{1}, \ldots, t_{i+k}\right)$,

we shall have an analytic functional $f\left[y\left(\boldsymbol{x}_{0}^{\prime}\right), y^{\prime}\left(\boldsymbol{x}_{0}\right)\right]$ which will be invariant under a transformation (1) whose kernel $K(x, s \mid a)$ is given by

$$
K(x, s \mid a)
$$

$$
=\sum_{i=1}^{\infty} \frac{a^{i}}{i !} \psi(s) e^{x / c} \int_{0}^{1} \int_{0_{(i-1)}}^{1} \ldots \int_{0}^{1} e^{1 \frac{1}{c_{j}-1} \sum_{j=1}^{t_{j}}} \prod_{l=1}^{i-1} \psi\left(t_{l}\right) d t_{1} \ldots d t_{i-1} .
$$

4. Example. We here give an easy example in which the direct verification by means of the finite transformation is very simple. Let us suppose that

$$
\delta y(x)=\left[e^{x} \int_{0}^{1} s y(s) d s\right] \delta a
$$

is the given infinitesimal transformation, i. e., that $H(x, s)$ $=s e^{x}$. By means of an easy calculation, the finite transformation may be written in the form

$$
y_{1}(x)=y(x)+\left(e^{a}-1\right) e^{x} \int_{0}^{1} s y(s) d s
$$

i. e., $K(x, s \mid a)=\left(e^{a}-1\right) e^{x} s$. Let us take for initial condition $f\left[y\left(\boldsymbol{\tau}_{0}^{\prime}\right), 0\right] \equiv F\left[y\left(\boldsymbol{\tau}_{0}^{\prime}\right)\right]$

$$
\equiv f_{00}+\int_{0}^{1} f_{10}\left(t_{1}\right) y\left(t_{1}\right) d t_{1}+\frac{1}{2 !} \int_{0}^{1} d t_{2} \int_{0}^{1} f_{20}\left(t_{1}, t_{2}\right) y\left(t_{1}\right) y\left(t_{2}\right) d t_{1} .
$$

Then the functional $f\left[y\left(\boldsymbol{\tau}_{0}^{\prime}\right), y^{\prime}\left(\boldsymbol{\tau}_{0}^{\prime}\right)\right]$ given by

$$
\begin{aligned}
& f\left[y\left(\boldsymbol{\tau}_{0}^{\prime}\right), y^{\prime}\left(\boldsymbol{\tau}_{0}^{\prime}\right)\right]=f_{00}+\int_{0}^{1} f_{10}\left(t_{1}\right)\left[y\left(t_{1}\right)-y^{\prime}\left(t_{1}\right)\right] d t_{1} \\
& +\frac{1}{2 !}\left[\int_{0}^{1} d t_{2} \int_{0}^{1} f_{20}\left(t_{1}, t_{2}\right)\left\{y\left(t_{1}\right) y\left(t_{2}\right)-2 y\left(t_{1}\right) y^{\prime}\left(t_{2}\right)+y^{\prime}\left(t_{1}\right) y^{\prime}\left(t_{2}\right)\right\}\right] d t
\end{aligned}
$$

is invariant under (20).

The Rice Institute 